\title{
INELASTIC LIGHT SCATTERING AND THE CORRELATED METAL-INSULATOR TRANSITION*
}

\author{
J.K. FREERICKS \\ Department of Physics, Georgetown University, Washington, DC 20057, USA \\ T.P. Devereaux \\ Department of Physics, University of Waterloo, Canada
}

AND R. Bulla

Theoretische Physik III, Elektronische Korrelationen und Magnitismus Institut für Physik, Universität Augsburg

86135 Augsburg, Germany

(Received July 10, 2002)

\begin{abstract}
Electronic Raman scattering has been employed to examine a number of different correlated insulators, including the high-temperature superconductors, Kondo insulators (like FeSi), and intermediate-valence compounds (like $\mathrm{SmB}_{6}$ ). The experimental data all share a number of common features: in the $\mathrm{B}_{1 g}$ channel (crossed polarizers) one finds (i) a sudden onset of low energy spectral weight transfered from a higher charge-transfer peak, which rapidly increases as $T$ increases; (ii) the appearance of an isosbestic point (where the Raman response is independent of $T$ ) separating the regions where the spectral weight shifts; and (iii) a large ratio of the spectral range over which spectral weight increases as $T$ increases (representative of the charge gap) to the onset temperature, where the gap appears to first open. We solve for the Raman response exactly using dynamical mean field theory for the Falicov-Kimball model and the Hubbard model. Our solutions illustrate all three of these experimental features. In addition, we calculate the inelastic light scattering from X-rays, which allows the photon to transfer both energy and momentum to the electronic charge excitations. We find that the charge transfer peak and the low energy peak both broaden and disperse through the Brillouin zone similar to what is seen in experiments in materials like $\mathrm{Ca}_{2} \mathrm{CuO}_{2} \mathrm{Cl}_{2}$.
\end{abstract}

PACS numbers: 78.30.-j, 71.30.+h, 74.72.-h, 75.20.Hr

${ }^{*}$ Presented at the International Conference on Strongly Correlated Electron Systems, (SCES 02), Cracow, Poland, July 10-13, 2002. 


\section{Introduction and experimental summary}

Light scattering has long been used as a bulk probe of the electronic charge excitations in solids. It is particularly interesting to study strongly correlated metals and insulators, since their charge dynamics are significantly renormalized and do not appear nearly free electron like. The most common form of light scattering is an elastic optical conductivity measurement, which usually proceeds by measuring the reflectivity and performing a Kramers-Kronig transformation to determine the optical conductivity (this normally requires extrapolations of the reflectivity for small and large frequency). Since the scattering is elastic, one adjusts the photon energy (color) to determine the energy dependence of the electronic charge excitations. Recently, there has been renewed interest in inelastic light scattering, named electronic Raman scattering when optical $(\boldsymbol{q}=0)$ light is used. Here one shines an intense monochromatic beam of light on the sample and studies the reflected light that emerges at a different energy, due to the inelastic scattering from the electronic charge excitations. By employing polarizers on the incident and reflected light, one can project the scattering onto different symmetry channels, thereby probing charge excitations of different symmetries. The three most common symmetries chosen are $A_{1 g}$ which has the full symmetry of the lattice (is $s$-like), $B_{1 g}$ which is a d-like symmetry, and $B_{2 g}$ which is another $d$-like symmetry. In addition, there have been a number of recent experiments probing inelastic X-ray scattering in correlated insulators. Here the photon can exchange both momentum and energy with the electronic charge excitations. These experiments usually require a resonant enhancement of the scattered signal by tuning the X-ray energy to lie close to a core edge transition.

Experimental electronic Raman scattering results on correlated insulators are plotted in figure 1. Each of these experimental systems share common features: (i) there is a sudden onset of low energy spectral weight transfered from a higher charge-transfer peak, which rapidly increases as $T$ increases; (ii) there is an isosbestic point (where the Raman response is independent of $T$ ) separating the regions where the spectral weight shifts; and (iii) the ratio of the spectral range over which spectral weight increases as $T$ increases (representative of the charge gap) to the onset temperature, where the gap appears to first open is much larger than the weak-coupling value of 3.5. The top panel shows $\mathrm{SmB}_{6}$ [1], which has the added feature of developing a sharp peak at $130 \mathrm{~cm}^{-1}$ (that does not disperse in frequency) when the temperature is lower than $30 \mathrm{~K}$. The FeSi data is shown in the middle panel [2]. It displays the cleanest signature of these anomalous features. Note how the isosbestic point only develops at temperatures below $150 \mathrm{~K}$. The bottom panel shows smoothed data in the LSCO high-temperature su- 
perconductor [3] (other HTSC experiments have also been performed $[4,5]$ ). The isosbestic point is somewhat harder to see here (because of the noise in the data), but it does develop at about $2100 \mathrm{~cm}^{-1}$ as the temperature is lowered.

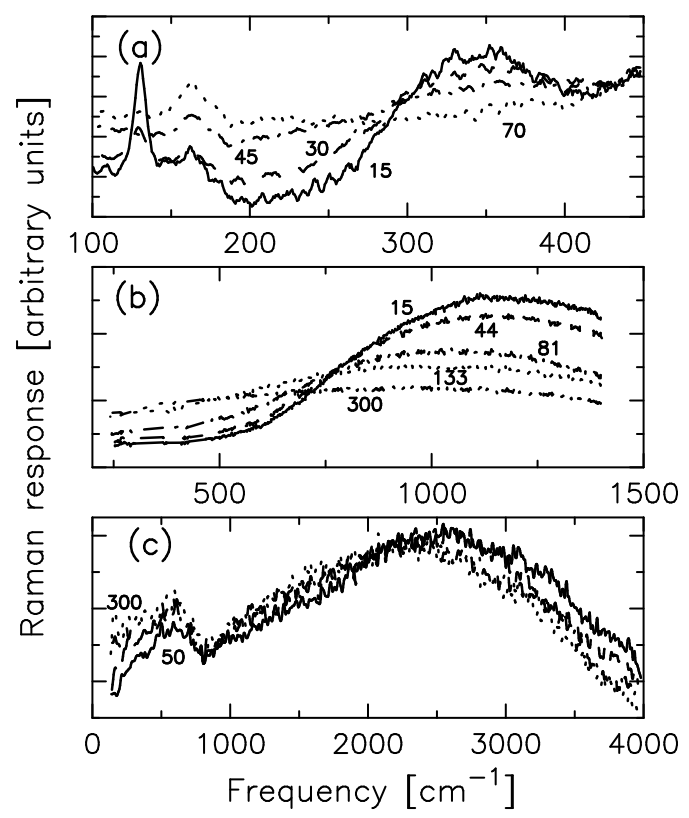

Fig. 1. Experimental $B_{1 g}$ Raman response for correlated materials (a) $\mathrm{SmB}_{6}$ [1]; (b) FeSi [2]; and (c) underdoped $\mathrm{La}_{2-x} \mathrm{Sr}_{x} \mathrm{CuO}_{4}$ [3] with $x=0.08$. All of the experimental data show the development of a low-temperature isosbestic point, which occurs due to the transfer of spectral weight from low energy to high energy as the temperature is lowered, indicating the proximity to the quantum-critical point of a metal-insulator transition. The individual curves are labeled by the temperature in $\mathrm{K}$ where the measurement was taken. In panel (c) only the high temperature $(300 \mathrm{~K})$ and the low temperature $(50 \mathrm{~K})$ are labeled. The two intermediate curves are at 100 and $200 \mathrm{~K}$, respectively.

In addition, resonant inelastic $\mathrm{X}$-ray scattering experiments have been performed on insulating compounds like $\mathrm{La}_{2} \mathrm{CuO}_{4}$ and $\mathrm{Sr}_{2} \mathrm{CuO}_{2} \mathrm{Cl}_{2}$ [6], $\mathrm{Ca}_{2} \mathrm{CuO}_{2} \mathrm{Cl}_{2}$ [7], $\mathrm{NaV}_{2} \mathrm{O}_{5}$ [8], $\mathrm{Nd}_{2} \mathrm{CuO}_{4}$ [9], and $\mathrm{Sr}_{2} \mathrm{CuO}_{3}$ and $\mathrm{SrCuO}_{2}$ [10]. These experiments require a tuning of the photon energy to lie close to the $\mathrm{Cu} \mathrm{K}$ or $\mathrm{V} L_{3}$ edge in order to get a large enough inelastic scattering signal. They then can be scanned through momentum space to examine the frequency and momentum dependent charge excitations in a correlated insulator. We concentrate here on the $\mathrm{Ca}_{2} \mathrm{CuO}_{2} \mathrm{Cl}_{2}$ data [7]. As a function 


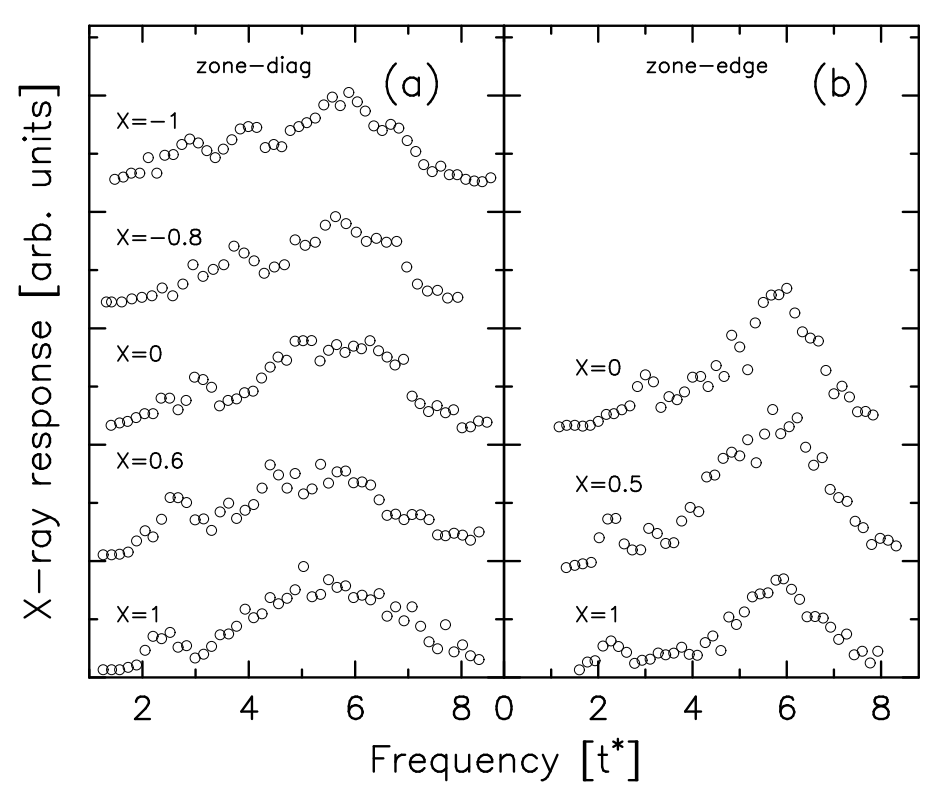

Fig. 2. Experimental resonant inelastic X-ray scattering in $\mathrm{Ca}_{2} \mathrm{CuO}_{2} \mathrm{Cl}_{2}$ [7] along (a) the zone diagonal and (b) the zone edge. The label $X$ is defined to be $X=$ $\left(\cos \boldsymbol{q}_{x}+\cos \boldsymbol{q}_{y}\right) / 2$ for photon scattering that transfers momentum $\boldsymbol{q}$.

of momentum one finds a charge transfer peak and a lower energy peak (these experiments use a linear polarizer for the incident light, so different symmetry channels are mixed together and the temperature is held at room temperature). As one scans through the Brillouin zone, the charge transfer peak hardly disperses, while the low-energy peak shows significant dispersion which tracks well with the parameter $X=\left(\cos \boldsymbol{q}_{x}+\cos \boldsymbol{q}_{y}\right) / 2$. It is hard to say what happens to the width of the peaks as one scans through the Brillouin zone, as the data is too noisy (because of the low intensity of the inelastic scattered signal). Note as well, the results for $X=1$ are not identical along the zone edge and zone diagonal because the use of polarizers projects onto different mixtures of the symmetry channels (these experiments have a fixed relation between the polarization of the electric field and the transferred momentum).

\section{Theoretical formalism}

We will examine two different model systems here: the Hubbard model [11] and the Falicov-Kimball model [12]. The Hubbard Hamiltonian [11] contains two terms: the electrons can hop between nearest neighbors (with 
hopping integral $t^{*} /(2 \sqrt{d})$ on a $d$-dimensional hypercubic lattice [13]), and they interact via a screened Coulomb interaction $U_{H}$ when they sit on the same site. All energies are measured in units of $t^{*}$. The Hamiltonian is

$$
H_{\mathrm{Hub}}=-\frac{t^{*}}{2 \sqrt{d}} \sum_{\langle i, j\rangle, \sigma} c_{i \sigma}^{\dagger} c_{j \sigma}+U_{H} \sum_{i} n_{i \uparrow} n_{i \downarrow},
$$

where $c_{i \sigma}^{\dagger}\left(c_{i \sigma}\right)$ is the creation (annihilation) operator for an electron at lattice site $i$ with spin $\sigma$ and $n_{i \sigma}=c_{i \sigma}^{\dagger} c_{i \sigma}$ is the electron number operator. We adjust a chemical potential $\mu$ to fix the average filling of the electrons to half filling $\left(\mu=U_{H} / 2\right)$.

The Falicov-Kimball Hamiltonian contains two types of electrons: itinerant band electrons and localized ( $c$ or $f$ ) electrons. The band electrons can hop between nearest neighbors in the same way as in the Hubbard model and they interact via a screened Coulomb interaction with the localized electrons (that is described by an interaction strength $U_{\mathrm{FK}}$ between electrons that are located at the same lattice site). The Hamiltonian is

$$
H_{\mathrm{FK}}=-\frac{t^{*}}{2 \sqrt{d}} \sum_{\langle i, j\rangle} c_{i}^{\dagger} c_{j}+E_{f} \sum_{i} w_{i}-\mu \sum_{i} c_{i}^{\dagger} c_{i}+U_{\mathrm{FK}} \sum_{i} c_{i}^{\dagger} c_{i} w_{i}
$$

where $c_{i}^{\dagger}\left(c_{i}\right)$ is the spinless conduction electron creation (annihilation) operator at lattice site $i$ and $w_{i}=0$ or 1 is a classical variable corresponding to the localized $f$-electron number at site $i$. We will adjust both $E_{f}$ and $\mu$ so that the average filling of the $c$-electrons is $1 / 2$ and the average filling of the $f$-electrons is $1 / 2\left(\mu=U_{\mathrm{FK}} / 2\right.$ and $\left.E_{f}=0\right)$. Note that if we do not allow the down-spin electrons in the Hubbard model to hop, then we get the spinless Falicov-Kimball model.

We focus here on nonresonant inelastic light scattering. In this case, the photon-electron vertex function does not depend on the photon frequency. It corresponds to the generic picture of light scattering, but is unable to predict additional properties associated with resonant enhancements of signals, which are often seen experimentally. We use the Kubo formula to relate the response function to the corresponding current-current correlation function. The currents for the different symmetry sectors each take the following form

$$
j_{a}(\boldsymbol{q})=\sum_{\sigma} \sum_{\boldsymbol{k}} \gamma_{a}(\boldsymbol{k}+\boldsymbol{q} / 2) c_{\boldsymbol{k}+\boldsymbol{q}_{\sigma}}^{\dagger} c_{\boldsymbol{k}_{\sigma}},
$$

with $\gamma_{a}(\boldsymbol{k})$ the corresponding current vertex function and the sum over spin needed only for the Hubbard model. For conventional Raman scattering, we 
work with optical light so $\boldsymbol{q} \rightarrow 0$, but the finite- $\boldsymbol{q}$ case is needed for X-ray scattering. The inelastic light scattering vertex is

$$
\gamma_{a}(\boldsymbol{k}+\boldsymbol{q} / 2)=\sum_{\alpha \beta} e_{\alpha}^{I} \frac{\partial^{2} \varepsilon(\boldsymbol{k}+\boldsymbol{q} / 2)}{\partial \boldsymbol{k}_{\alpha} \partial \boldsymbol{k}_{\beta}} e_{\beta}^{O *},
$$

where the $e$ vectors denote the incoming and outgoing photon polarizations and $\varepsilon(\boldsymbol{k})$ is the noninteracting bandstructure (we examine a hypercubic lattice here).

The Dyson equation for the current-current correlation function takes the form given in Fig. 3. Note that there are two coupled equations illustrated in Figs. 3 (a) and (b); these equations differ by the number of $\gamma_{a}$ factors in them. The irreducible vertex function $\Gamma$ is the dynamical charge vertex [14] which takes the form

$$
\Gamma\left(i \omega_{m}, i \omega_{n} ; i \nu_{l \neq 0}\right)=\delta_{m n} \frac{1}{T} \frac{\Sigma_{m}-\Sigma_{m+l}}{G_{m}-G_{m+l}} .
$$

on the imaginary axis for the Falicov-Kimball model $\left[i \omega_{m}=i \pi T(2 m+1)\right.$ is the Fermionic Matsubara frequency and $i \nu_{l}=2 i \pi T l$ is the Bosonic Matsubara frequency]. Here $\Sigma_{m}=\Sigma\left(i \omega_{m}\right)$ is the local self energy on the imaginary axis and $G_{m}=G\left(i \omega_{m}\right)$ is the local Green's function on the imaginary axis. An explicit expression for the irreducible charge vertex is not known for the Hubbard model, but it also possesses the full symmetry of the lattice. If the vertex factor $\gamma_{a}$ does not have a projection onto the full symmetry of the lattice, then there are no vertex corrections from the local dynamical charge vertex [15].
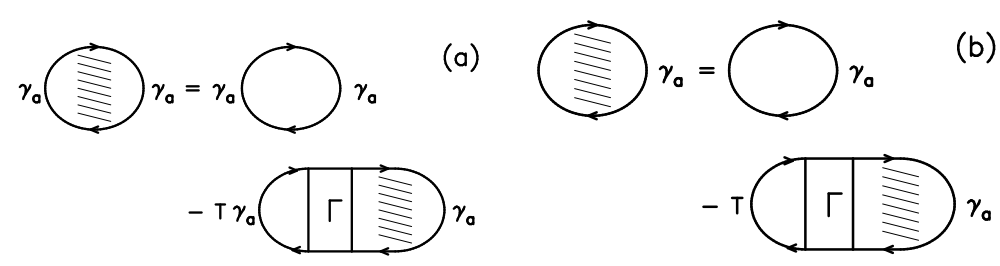

Fig. 3. Coupled Dyson equations for the inelastic light scattering current-current correlation functions described by the vertex function $\gamma_{a}$. Panel (a) depicts the Dyson equation for the interacting correlation function, while panel (b) is the supplemental equation needed to solve for the correlation function. The symbol $\Gamma$ stands for the local dynamical irreducible charge vertex given in Eq. (5) for the Falicov-Kimball model. In situations where there are no charge vertex corrections, the correlation function is simply given by the first (bare-bubble) diagram on the right-hand side of panel (a). 
There are traditionally three main symmetries considered in Raman scattering experiments: (i) $A_{1 g}$ which has the full symmetry of the lattice; (ii) $B_{1 g}$ which has a $d$-wave symmetry and (iii) $B_{2 g}$ which is another $d$-wave symmetry. Each symmetry is chosen by different polarizations for the incident and scattered light. If we sum over the $d$ pairs of polarizations, where $e^{I}=e^{O}$ and each vector points along each of the different Cartesian axes, then we have the $A_{1 g}$ sector. If we choose $e^{I}=(1,1,1, \ldots)$ and $e^{O}=(1,-1,1,-1, \ldots)$, then we have the $B_{1 g}$ sector. And if we choose $e^{I}=(1,0,1,0, \ldots)$ and $e^{O}=(0,-1,0,-1, \ldots)$ then we have the $B_{2 g}$ sector. If we have just nearest-neighbor hopping, then the $B_{2 g}$ response vanishes because $\gamma_{B_{2 g}}=0$. Following the form given in Eq. (4), we find $\gamma_{A_{1 g}}(\boldsymbol{q})=-\varepsilon(\boldsymbol{q})$ and $\gamma_{B_{1 g}}(\boldsymbol{q})=t^{*} \sum_{j=1}^{\infty} \cos \boldsymbol{q}_{j}(-1)^{j} / \sqrt{d}$.

A straightforward calculation, shows that the $B_{1 g}$ response has no vertex corrections on the zone diagonal $\boldsymbol{q}=(q, q, q, q, \ldots)$. Hence, the $B_{1 g}$ response is the bare bubble and can be determined in both the FalicovKimball model and the Hubbard model. The $A_{1 g}$ response everywhere and the $B_{1 g}$ response off of the zone diagonal, do have vertex corrections, and can only be determined for the Falicov-Kimball model. The calculation of each response function is straightforward, but tedious. One needs to first solve the coupled equations depicted in Fig. 3 on the imaginary axis and then perform the analytic continuation as in the Raman scattering case [16]. The end result is cumbersome and will not be given here. Instead we show the (bare-bubble) $B_{1 g}$ result on the zone diagonal

$$
\begin{aligned}
\chi_{B_{1 g}}(\boldsymbol{q}, \nu)= & \frac{i}{4 \pi} \int_{-\infty}^{\infty} d \omega\left\{f(\omega) \chi_{0}(\omega ; X, \nu)-f(\omega+\nu) \chi_{0}^{*}(\omega ; X, \nu)\right. \\
& \left.-[f(\omega)-f(\omega+\nu)] \tilde{\chi}_{0}(\omega ; X, \nu)\right\}
\end{aligned}
$$

with

$$
\begin{aligned}
\chi_{0}(\omega ; X, \nu)= & -\int_{-\infty}^{\infty} d \varepsilon \rho(\varepsilon) \frac{1}{\omega+\mu-\Sigma(\omega)-\varepsilon} \frac{1}{\sqrt{1-X^{2}}} \\
& \times F_{\infty}\left(\frac{\omega+\nu+\mu-\Sigma(\omega+\nu)-X \varepsilon}{\sqrt{1-X^{2}}}\right),
\end{aligned}
$$

and

$$
\begin{aligned}
\tilde{\chi}_{0}(\omega ; X, \nu)= & -\int_{-\infty}^{\infty} d \varepsilon \rho(\varepsilon) \frac{1}{\omega+\mu-\Sigma^{*}(\omega)-\varepsilon} \frac{1}{\sqrt{1-X^{2}}} \\
& \times F_{\infty}\left(\frac{\omega+\nu+\mu-\Sigma(\omega+\nu)-X \varepsilon}{\sqrt{1-X^{2}}}\right) .
\end{aligned}
$$


Here we have used the following notation: $f(\omega)=1 /[1+\exp (\omega / T)]$ is the Fermi-Dirac distribution, $\rho(\varepsilon)=\exp \left(-\varepsilon^{2}\right) / \sqrt{\pi}$ is the noninteracting density of states; $\Sigma(\omega)$ is the local self energy on the real axis; $X=\cos q$ for the zone-diagonal wavevector $\boldsymbol{q}=(q, q, q, \ldots, q)$; and $F_{\infty}(z)=\int d \varepsilon \rho(\varepsilon) /(z-\varepsilon)$ is the Hilbert transform of the noninteracting density of states. Techniques for finding the self energy for the Hubbard model [17] and the Falicov-Kimball model [18] have appeared elsewhere.

\section{Theoretical results}

We plot results for the inelastic X-ray scattering in the Hubbard model in Fig. 4. Three cases are considered: (a) a correlated metal, (b) a material undergoing a metal-insulator transition, and (c) a correlated insulator. The curves have been shifted vertically for clarity. The lowest set of curves $X=1$ correspond to Raman scattering with optical photons [19].

Consider first the correlated metal in panel (a). At $\boldsymbol{q}=0$, we see the development of a characteristic Fermi peak at low energy that narrows and moves towards $\nu=0$ as the temperature is lowered. As we move away from the zone center, the Fermi peak broadens and has its maximum lie at a finite frequency. This is exactly what one would expect, since the damping increases dramatically as scattering in the particle-hole continuum becomes possible (for larger $\boldsymbol{q}$ values). Note also that the charge-transfer peak has little $T$-dependence at $X=0$. In panel (b), we show the results for a system that undergoes a temperature-dependent metal-insulator transition at $T \approx 0.011$. Note how low-energy spectral weight is initially depleted as $T$ is lowered, but then returns as the system becomes more metallic. The temperature dependence is reduced as we move towards the zone corner. In addition, the isosbestic point disperses to higher energy as $\boldsymbol{q}$ is increased. Finally, there is a small low-energy peak that emerges at low- $T$, and is present with only slight dispersion in the Brillouin zone but with growing weight as the zone corner is approached. In panel (c), we plot results for a correlated insulator. Here all momenta allow the development of lowenergy spectral weight as $T$ increases and there is an isosbestic point, but the isosbestic point does not disperse with $\boldsymbol{q}$ anymore. Also, we see the broadening of the charge-transfer peak as we move toward the zone corner.

In Fig. 5 we plot the inelastic X-ray scattering at $U_{\mathrm{FK}}=2$ (a) for the $B_{1 g}$ channel along the zone diagonal, (b) for the $B_{1 g}$ channel along the "zone edge" [here we have $\boldsymbol{q}=(q, 0, q, 0, \ldots, q, 0)$ for $1 \leq X=(1+\cos q) / 2 \leq 0$ and $\boldsymbol{q}=(\pi, q, \pi, q, \ldots, \pi, q)$ for $0 \leq X=(-1+\cos q) / 2 \leq-1]$, and (c) for the $A_{1 g}$ channel along the zone diagonal. The correlation strength $U_{\mathrm{FK}}$ was chosen to be 2 , which is just on the insulating side of the metal-insulator transition. Note how the results are all identical at the $(\pi, \pi, \ldots, \pi)$ point. 


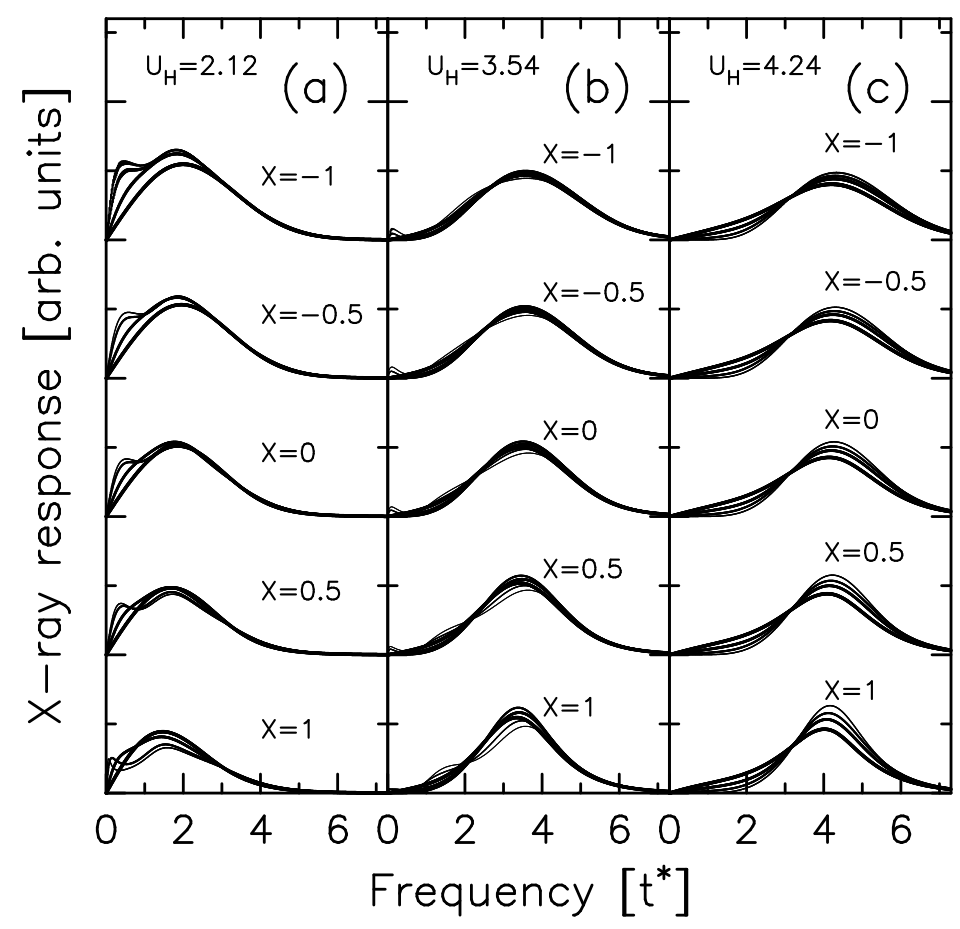

Fig. 4. Inelastic X-ray scattering response in the $B_{1 g}$ channel along the Brillouin zone diagonal for the half-filled Hubbard model on a hypercubic lattice. Panel (a) shows a correlated metal $(T=0.353,0.150,0.064,0.039)$, panel (b) shows a material that undergoes a metal insulator transition as a function of temperature $(T=0.282,0.172,0.039,0.009,0.003)$ and panel (c) shows a correlated insulator ( $T=0.566,0.424,0.283,0.071)$. The different thicknesses of the curves correspond to different temperatures (thinnest being the lowest temperature).

This occurs due to the local approximation. Any variation in the signal at the zone corner in different symmetry channels must be due to nonlocal many-body correlations. Note also how the $A_{1 g}$ results have no low-energy spectral weight for $\boldsymbol{q}=0$. The vertex corrections remove all remnants of the low-energy response here, but it enters for any finite value of $\boldsymbol{q}$. The main qualitative feature is that the charge-transfer peak broadens significantly as we move through the Brillouin zone, and the results along the zone edge are quite similar to those along the zone boundary, when plotted with the corresponding $X$-values. We can see a small amount of dispersion of the low-energy peak through the Brillouin zone, but it is not strong effect for when the gap in the insulator is small. Finally, the isosbestic point seen at $\boldsymbol{q}=0$ appears for all $\boldsymbol{q}$. 


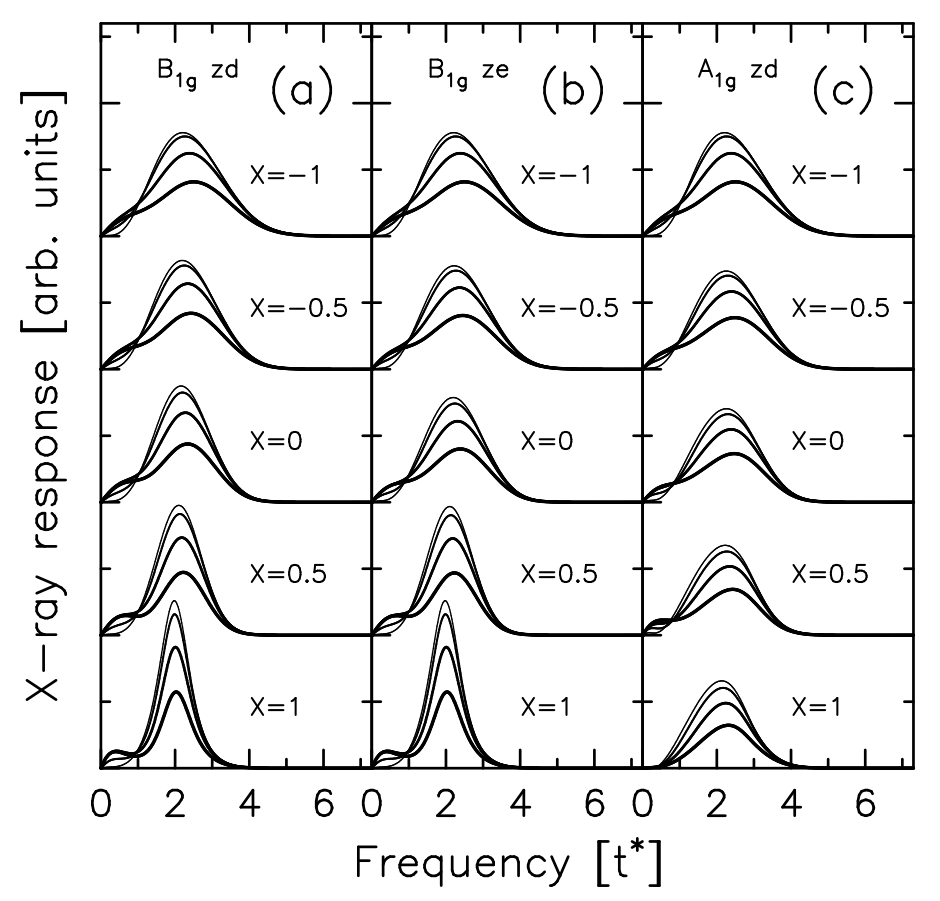

Fig. 5. Inelastic X-ray scattering response in the $B_{1 g}$ channel along (a) the Brillouin zone diagonal and (b) along the zone edge and (c) in the $A_{1 g}$ channel along the zone diagonal for the half-filled Falicov-Kimball model on a hypercubic lattice. The correlation strength is $U_{\mathrm{FK}}=2$ which is just on the insulating side of the metal-insulator transition for the FK model. The different curves correspond to different temperatures ranging from thickest to thinnest curve as follows: $T=1.0$, $0.5,0.25,0.1$.

We should also point out that all of the anomalous features seen in experiment for Raman scattering $(\boldsymbol{q}=0)$ are also seen here. Low-energy spectral weight emerges at low temperature in the $B_{1 g}$ channel but not the $A_{1 g}$ channel; there is an isosbestic point; and the ratio of twice the chargegap $\approx 1$ to the transition temperature $\approx 0.2$ is about 10 .

\section{Conclusions}

We have examined inelastic light scattering in correlated materials through the metal-insulator transition for two different models of electron correlations: the Hubbard model and the Falicov-Kimball model. In the Hubbard model we could only determine results that were not renormalized by the irreducible charge vertex (along the Brillouin zone diagonal), but 
we could determine results everywhere in the Falicov-Kimball model. We found a number of interesting features in the solutions including the presence of three well-known anomalies in the $\boldsymbol{q}=0$ Raman scattering case. In addition, for finite $\boldsymbol{q}$, we saw new features emerge including the following: the absence of symmetry dependence at the zone corner; the occurrence of isosbestic points throughout the Brillouin zone; and a generic broadening of the charge transfer peak as one moves from the zone center to the zone corner. Many of these latter results have not yet been seen in inelastic $\mathrm{X}$-ray scattering. We believe it would be quite interesting to examine inelastic X-ray scattering at different temperatures and with polarizers for both the incident and scattered light. We believe that a number of new and interesting features of charge excitations in correlated systems are likely to emerge if this can be accomplished.

We would like to thank L. Cooper, C. Irwin, R. Hackl, and Z. Hasan for sharing their experimental data with us and we would like to thank Z.-X. Shen and M. Klein for valuable discussions. J.K.F. acknowledges support from the NSF under grant number DMR-9973225. T.P.D. acknowledges support by NSERC and R.B. acknowledges support by the Deutsche Forschungsgemeinschaft through the Sonderforschungsbereich 484 .

\section{REFERENCES}

[1] P. Nyhus, S.L. Cooper, Z. Fisk, J. Sarrao, Phys. Rev. B52, R14308 (1995); Phys. Rev. B55, 12488 (1997).

[2] P. Nyhus, S.L. Cooper, Z. Fisk, Phys. Rev. B51, R15626 (1995).

[3] T. Katsufuji, Y. Tokura, S. Uchida, Phys. Rev. B48, 16131 (1993); X.K. Chen, J.G. Naeini, K.C. Hewitt, J.C. Irwin, R. Liang, W.N. Hardy, Phys. Rev. B56, R513 (1997); J.G. Naeini, X.K. Chen, J.C. Irwin, M. Okuya, T. Kimura, K. Kishio, Phys. Rev. B59, 9642 (1999).

[4] M. Opel, R. Nemetschek, C. Hoffmann, R. Philipp, P.F. Müller, R. Hackl, I. Tütto, A. Erb, B. Revaz, E. Walker, H. Berger, L. Forró, Phys. Rev. B61, 9752 (2000); F. Venturini, M. Opel, T.P. Devereaux, J.K. Freericks, I. Tütto, B. Revaz, E. Walker, H. Berger, L. Forró, R. Hackl, Phys. Rev. Lett. 89, 107003 (2002).

[5] M. Rübhausen, O.A. Hammerstein, A. Bock, U. Merkt, C.T. Rieck, P. Guptasarma, D.G. Hinks, M.V. Klein, Phys. Rev. Lett. 82, 5349 (1999); S. Sugai, T. Hosokawa, Phys. Rev. Lett. 85, 1112 (2000).

[6] P. Abbamonte, C.A. Burns, E.D. Isaacs, P.M. Platzman, L.L. Miller, S.W. Cheong, M.V. Klein, Phys. Rev. Lett. 83, 860 (1999).

[7] M.Z. Hasan, E.D. Isaacs, Z.-X. Shen, L.L. Miller, K. Tsutsui, T. Tohyama, S. Maekawa, Science 288, 1811 (2000); M.Z. Hasan, E.D. Isaacs, Z.-X. Shen, L.L. Miller, Physica C 364-365, 618 (2001). 
[8] G.P. Zhang, T.A. Callcott, G.T. Woods, L. Lin, Phys. Rev. Lett. 88, 077401 (2002).

[9] K. Hämäläinen, J.P. Hill, S. Huotari, C.-C. Kao, L.E. Berman, A. Kotani, T. Idé, J.L. Peng, R.L. Greene, Phys. Rev. B61, 1836 (2000).

[10] M.Z. Hasan, P.A. Montano, E.D. Isaacs, Z.-X. Shen, H. Eisaki, S.K. Sinha, Z. Islam, N. Motoyama, S. Uchida, Phys. Rev. Lett. 88, 177403 (2002).

[11] J.C. Hubbard, Proc. R. Soc. Lond., Ser. A 276, 238 (1963).

[12] L.M. Falicov, J.C. Kimball, Phys. Rev. Lett. 22, 997 (1969).

[13] W. Metzner, D. Vollhardt, Phys. Rev. Lett. 62, 324 (1989).

[14] A.M. Shvaika, Physica C 341-348, 177 (2000); J.K. Freericks, P. Miller, Phys. Rev. B62, 10022 (2000); A.M. Shvaika, J. Phys. Studies 5, 349 (2001).

[15] A. Khurana, Phys. Rev. Lett. 64, 1990 (1990).

[16] J.K. Freericks, T.P. Devereaux, Condens. Mat. Phys. 4, 149 (2001); Phys. Rev. B64, 125110 (2001).

[17] R. Bulla, Phys. Rev. Lett. 83, 136 (1999).

[18] U. Brandt, C. Mielsch, Z. Phys. B75, 365 (1989); V. Zlatić, J.K. Freericks, R. Lemański, G. Czycholl, Philos. Mag. B81, 1443 (2001).

[19] J.K. Freericks, T.P. Devereaux, R. Bulla, Acta Phys. Pol. B 32, 3219 (2001); Phys. Rev. B64, 233114 (2001). 